\title{
Les éconduits de la Science : les demandes de missions lointaines rejetées par le ministère de l'Instruction publique (1842-1900)
}

Turned away by Science: Applications for Overseas Missions Rejected by the Ministère de l'Instruction Publique (1842-1900)

Von der Wissenschaft abgewiesen : Die vom Ministerium abgelehnten Anträge auf Überseeeinsätze (1842-1900)

\section{Stéphanie Soubrier}

\section{(2) OpenEdition}

\section{Journals}

Édition électronique

URL : https://journals.openedition.org/rh19/5962

DOI : 10.4000/rh19.5962

ISSN : $1777-5329$

Éditeur

La Société de 1848

Édition imprimée

Date de publication : 26 décembre 2018

Pagination : 75-90

ISSN : 1265-1354

Référence électronique

Stéphanie Soubrier, «Les éconduits de la Science : les demandes de missions lointaines rejetées par le ministère de I'Instruction publique (1842-1900) », Revue d'histoire du XIXe siècle [En ligne], 57 | 2018, mis en ligne le 26 décembre 2020, consulté le 21 janvier 2022. URL : http://journals.openedition.org/ rh19/5962 ; DOl : https://doi.org/10.4000/rh19.5962 


\section{STÉPHANIE SOUBRIER}

\section{Les éconduits de la Science: les demandes de missions lointaines rejetées par le ministère de l'Instruction publique (1842-1900)}

Les nombreuses demandes de missions scientifiques et littéraires reçues par le ministère de l'Instruction publique au XIX' siècle, qui sont conservées dans la série F17 des archives nationales, ressemblent parfois à s'y méprendre à des lettres d'amour : le botaniste Louis Crié y professe «l'amour le plus désintéressé de la science», tandis qu'Edmond Durighello, fils d'un viceconsul de France en Syrie, se dit «épris des fouilles» et atteint par la passion de l'archéologie, "bien plus puissante que toutes les autres passions». Ces lettres reflètent l'apparition, au sein de la société française du XIX ${ }^{\mathrm{e}}$ siècle, d'un "désir nouveau de savoir ${ }^{1}$ ", qui se manifeste notamment par l'essor des publications vulgarisatrices, la multiplication des sociétés savantes et l'engouement pour les pratiques scientifiques amateures ${ }^{2}$. Les dossiers reçus par le ministère de l'Instruction publique fournissent des données supplémentaires pour saisir l'ampleur et les ressorts de cette nouvelle libido sciendi. Créé en 1828, le ministère organise et subventionne dès les années 1840 des missions scientifiques en France ou à l'étranger. Ce rôle s'accroît à partir du 21 janvier 1874, date à laquelle est constituée une commission des missions et des voyages scientifiques et littéraires, chargée d'examiner les demandes de mission déposées par des particuliers, d'encadrer les voyageurs et d'exploiter les fruits de leurs travaux. Entre 1840 et 1914, le ministère a attribué 1102 missions gratuites et 1205 missions payées. Seules ces dernières ont été étudiées par l'historien Jean-Christophe Bourquin ${ }^{3}$, qui a mis en lumière l'importance des liens entre l'État et les voyageurs savants au XIX'e siècle.

1. Alain Corbin, Les conférences de Morterolles, hiver 1895-1896. À l'écoute d'un monde disparu, Paris, Flammarion, 2013, p. 14.

2. Voir notamment Hervé Guillemain et Nathalie Richard, "Towards a Contemporary Historiography of Amateurs in Science (18th-20th Century)", Gesnerus, vol. 73, n 2, 2016, p. 201237; Dominique Lejeune, Les sociétés de géographie en France, dans le monvement social et intellectuel $d u$ XIXe siècle, thèse de doctorat d'histoire, Université Paris 10, 1987.

3. Jean-Christophe Bourquin, L'État et les voyageurs savants. Légitimités individuelles et volontés politiques. Les missions du ministère de l'Instruction publique, 1840-1914, thèse d'histoire sous la direction d'Antoine Prost, Paris 1, 1993. 
Si le ministère de l'Instruction publique a fait beaucoup d'heureux, il a également brisé bien des cœurs, en refusant 652 candidatures. Ce sont ces dossiers, largement méconnus, que cet article se propose d'étudier à travers un échantillon plus restreint de 292 demandes de mission outre-mer ${ }^{4}$. Cellesci apparaissent comme un prolongement du lien établi dès le XVIII siècle par les philosophes encyclopédistes entre le voyage et la connaissance scientifique ${ }^{5}$. Ce lien se matérialise notamment dans les nombreuses demandes de missions d'exploration, qui impliquent à la fois un déplacement lointain en territoire inconnu ou mal connu et la mise au jour de données susceptibles de faire progresser les différentes disciplines scientifiques : à la phase initiale de découverte, opérée par des explorateurs solitaires ayant pour principal objectif de «faire beaucoup de chemin et de passer là où nul n'avait mis le pied " succède, à partir de la fin des années 1880 , l'ère de la colonisation, durant laquelle la science est souvent présentée par les acteurs de la conquête comme l'un des piliers de la «mission civilisatrice ${ }^{7}$. Dans les dernières décennies du $\mathrm{XIX}^{\mathrm{e}}$ siècle, la figure de l'explorateur acquiert une popularité inédite, alors même que l'exploration se confond progressivement avec le récit de l'appropriation symbolique et effective du monde par les puissances coloniales européennes ${ }^{8}$. On peut ainsi s'interroger sur l'émergence d'une dimension impériale de la libido sciendi, qui serait contemporaine de l'expansion coloniale du XIX ${ }^{\mathrm{e}}$ siècle et en épouserait les grandes inflexions chronologiques, depuis les premières explorations jusqu'à la mise en place de la domination coloniale, et sur ses modalités particulières, qui la distinguent des grands voyages d'exploration du siècle précédent.

4. Certains de ces dossiers ne figurent pas dans l'inventaire établi par M.-E. Antoine et S. Olivier, Inventaire des papiers de la division des Sciences et Lettres du ministère de l'Instruction publique et des services qui en sont issus (sous-série F17), tome II, Archives nationales, 1981. À l'inverse, certains dossiers de demandes de mission qui figurent dans l'inventaire sont incommunicables ou manquants. On a étudié l'ensemble des dossiers conservés aux Archives nationales sous les cotes F17 29331 à F17 30142 et exclu de l'échantillon les dossiers conservés dans les cotes F17 17265 à F17 17294, qui concernent dans leur immense majorité des demandes postérieures à 1900. On a exclu de l'échantillon les 360 demandes concernant l'Europe.

5. Marie-Noëlle Bourguet et Christian Licoppe, «Voyages, mesures et instruments : une nouvelle expérience du monde au siècle des Lumières", Annales. Histoire, sciences sociales, n ${ }^{\circ}$, 1997, p. 11151151 ; Justin Stagl, A History of Curiosity, 1550-1800, Philadelphie, Harwood Academic Publishers, 1995.

6. Charles Maunoir, Le Tour du Monde, 1888, I, p. 417, cité par Numa Broc, «Les explorateurs français du XIX" siècle reconsidérés", Revue française d'histoire d'outre-mer, nº 256, 1982, p. 238.

7. De nombreuses études ont cependant démontré que les rapports entre science et colonisation au XIX ${ }^{e}$ siècle ne peuvent être réduits à une subordination de la première à la deuxième. $C f$. notamment Talal Asad (dir), Anthropology and the Colonial Encounter, New York, Humanities Press, 1985 (1973); Daniel Nordman et Jean-Pierre Raison (dir), Sciences de l'homme et conquête coloniale : constitution et usages des sciences humaines en Afrique, XIXé-XXe siècles, Paris, Éditions rue d'Ulm, 1980; Emmanuelle Sibeud, Une science impériale pour l'Afrique? La construction des savoirs africanistes en France, 18781930, Paris, Éditions de l'EHESS, 2002 ; Pierre Singaravélou (dir), L'empire des géographes. Géographie, exploration et colonisation, XIX ${ }^{e}-X X^{e}$ siècle, Paris, Belin, 2008; Li Chuan Tai, L'anthropologie française entre sciences coloniales et décolonisation (1880-1960), Paris, Éditions de la SFHOM, 2011.

8. Isabelle Surun, "Les figures de l'explorateur dans la presse du XIX siècle», Le Temps des médias, nº 8, 2007, p. 72. 
En s'intéressant aux échecs de la libido sciendi plutôt qu'à ses réussites, nous postulons ici, dans le sillage des perspectives ouvertes à la fin des années 1970 par la sociologie des connaissances de David Bloor' et de l'école d'Edimbourg, qu'une telle démarche ne condamne pas l'historien à une histoire anecdotique et qu'elle ne se cantonne pas à une approche par les marges. Accorder la même attention aux pétitionnaires malheureux qu'aux chargés de missions permet ainsi de réinscrire les connaissances scientifiques dans leur contexte d'élaboration en s'affranchissant d'une perspective internaliste, téléologique et anachronique. L'attrait exercé par les subsides de la commission permet sans doute d'expliquer la surreprésentation, dans l'échantillon, d'individus marginaux (escrocs, imposteurs, etc.) et, plus largement, une modification du profil sociologique qui avait été dégagé par Jean-Christophe Bourquin : on trouve ainsi parmi les pétitionnaires malheureux un coiffeur, plusieurs employés de commerce, des comptables, des secrétaires, des soldats du rang et toute une cohorte d'individus à la recherche d'un emploi ou d'une nouvelle vie. Les dossiers de mission, documents standardisés produits par une institution à la longévité et à la stabilité exceptionnelles, se prêtent particulièrement bien à un traitement quantitatif mis au service d'une histoire sociale des savoirs ${ }^{10}$ et permettent de tracer les contours d'un groupe très hétérogène, d'un point de vue géographique, social et culturel. Si la faiblesse relative de l'échantillon restreint l'échelle des conclusions qu'il est possible d'en tirer, les dossiers de mission refusés permettent en revanche d'enrichir la connaissance des ressorts individuels de la libido sciendi. En effet, loin de se présenter comme des documents administratifs arides, les lettres de demandes de mission, souvent longues et introspectives, constituent des sources précieuses pour aborder d'une manière nouvelle les représentations attachées à la connaissance scientifique et à l'exploration dans la France du $\mathrm{XIX}^{\mathrm{e}}$ siècle, et ce y compris hors des cercles académiques.

Enfin, les documents produits par les membres de la commission, qu'il s'agisse des comptes rendus de projets, de la correspondance qu'ils suscitent ou encore des copies des lettres de refus adressées aux demandeurs, donnent à voir les logiques sociales à l'œuvre dans la régulation de la libido sciendi et la mise en place de mécanismes d'exclusion et de marginalisation de certaines catégories d'individus et de projets, qui contribuent à la redéfinition constante des frontières de la science légitime.

9. David Bloor, Knowledge and Social Imagery, Londres, Routledge, 1976.

10. Lewis Pyenson, "Who the Guys Were' : Prosopography in the History of Science", History of Science, 25, 1977, p. 155-188. 


\section{Portrait statistique Des Demandeurs De Missions}

L'échantillon étudié comprend 292 individus statistiques et 273 individus réels $s^{11}$, aux profils extrêmement variés, qui révèlent, en filigrane, les logiques de sélection mises en œuvre par la commission. Ce sont quasiment tous des hommes - on ne compte que trois femmes ${ }^{12}$ - âgés de 18 à 66 ans. Leur lieu de résidence révèle le handicap dont souffrent les demandeurs provinciaux : en effet, $34 \%$ de demandes ont été envoyées depuis la province, mais seuls $18,6 \%$ des chargés de mission sont provinciaux. Les 30 demandes envoyées depuis l'étranger témoignent quant à elles du rayonnement de la commission, qui fonctionne comme un pôle majeur à l'échelle internationale. La moitié provient d'individus résidant dans les principales villes des colonies françaises, avec une prédominance de l'Algérie, d'où sont expédiées huit demandes de mission.

L'analyse des métiers exercés par les candidats illustre la grande diversité socio-professionnelle des amateurs de science au XIX ${ }^{\text {e }}$ siècle. Parmi les 292 dossiers déposés, 23,6\% l'ont été par des individus dont la science ne constitue pas l'activité principale ${ }^{13}$. Ces amateurs comprennent des avocats, des ingénieurs, des petits employés, des journalistes, ainsi que cinq chefs d'entreprise et un photographe, et ne représentent que 1,8\% des missions subventionnées par le ministère. Il ne faudrait pas pour autant en conclure que le ministère réserve ses subventions aux savants professionnels. Comme le rappelle Jean-Christophe Bourquin, «les pratiques savantes du XIX ${ }^{e}$ siècle sont plus ouvertes aux amateurs que celles de notre temps $»^{14}$ et les amateurs lettrés ayant reçu une formation intellectuelle constituent près de $13 \%$ des chargés de mission. Cette relative ouverture s'explique par la division du travail scientifique, dont une part importante consiste à réunir, sur les différents terrains de recherche, des données et des spécimens qui sont ensuite analysés et étudiés par les savants de métropole ${ }^{15}$. La commission, encouragée en cela par la réussite célèbre d'amateurs comme René Caillié ou Édouard Foa, considère que n'importe quel voyageur bien préparé et muni d'instructions est à même de fournir des données qui feront progresser les connaissances scientifiques. Toutefois, elle semble privilégier ceux que leur métier prédispose à la pratique de l'observation scientifique et du recueil de données : les médecins, que la pratique clinique familiarise avec les données chiffrées, ainsi que les architectes et les dessinateurs, dont on valorise les capacités techniques, constituent 7,1\% des chargés de mission. Mais ils sont

11. Dix-sept pétitionnaires ont demandé plusieurs missions et certains ont formulé une demande de mission pour deux personnes.

12. Archives nationales (AN), voir les dossiers de Dorothée Chellier-Castelli (F17 2948), Amélie Ernst (F17 2960) et Anne Levinck (F17 2984 2).

13. Pour un portrait détaillé de ce groupe très hétérogène, et les problèmes de définition qu'il soulève $c f$. Hervé Guillemain et Nathalie Richard, art.cit.

14. Jean-Christophe Bourquin, L'État et les voyageurs savants..., op. cit, p. 55.

15. Voir Emmanuelle Sibeud, Une science impériale pour l'Afrique?, op. cit. 
Profession des demandeurs de mission

\begin{tabular}{|c|c|c|c|c|}
\hline Profession & $\begin{array}{c}\text { Nombre de } \\
\text { demandeurs de } \\
\text { mission }\end{array}$ & $\begin{array}{c}\% \text { des demandeurs } \\
\text { de mission }\end{array}$ & $\begin{array}{c}\text { Nombre de } \\
\text { chargés de mission }\end{array}$ & $\begin{array}{c}\% \text { des chargés de } \\
\text { mission }^{1}\end{array}$ \\
\hline $\begin{array}{l}\text { Personnels des } \\
\text { facultés }\end{array}$ & 7 & 2,4 & 210 & 17,4 \\
\hline $\begin{array}{l}\text { Personnels des grands } \\
\text { établissements }\end{array}$ & 9 & 3,1 & 257 & 21,3 \\
\hline $\begin{array}{l}\text { Archivistes et } \\
\text { bibliothécaires }\end{array}$ & 2 & 0,7 & 116 & 9,6 \\
\hline $\begin{array}{l}\text { Professeurs de lycée et } \\
\text { d'autres institutions }\end{array}$ & 17 & 5,9 & 84 & 7 \\
\hline $\begin{array}{l}\text { Employés des } \\
\text { ministères }\end{array}$ & 36 & 12,3 & 121 & 10 \\
\hline Militaires et marins & 27 & 9,2 & 30 & 2,5 \\
\hline $\begin{array}{l}\text { Médecins de la marine } \\
\text { et médecins militaires }\end{array}$ & 5 & 1,7 & 11 & 0,9 \\
\hline Médecins & 25 & 8,6 & 46 & 3,8 \\
\hline $\begin{array}{l}\text { Architectes, peintres et } \\
\text { dessinateurs }\end{array}$ & 11 & 3,8 & 29 & 2,4 \\
\hline Ecclésiastiques & 7 & 2,4 & 19 & 1,6 \\
\hline $\begin{array}{l}\text { Dilettantes } \\
\text { Dont : }\end{array}$ & 32 & 11 & 260 & 21,6 \\
\hline $\begin{array}{l}\text { Amateurs lettrés: } \\
\text { Explorateurs et } \\
\text { voyageurs : }\end{array}$ & 23 & 7,9 & 154 & 12,8 \\
\hline Autres & 69 & 23,6 & 22 & 1,8 \\
\hline Profession inconnue & 45 & 15,4 & 6 & 0,5 \\
\hline
\end{tabular}

1. Les données concernant les chargés de mission sont tirées de Jean-Christophe Bourquin, L'État et les voyageurs savants..., op. cit.

plus nombreux parmi les candidats refusés (14,1\%), la commission agissant ici comme une instance régulatrice de leur libido sciendi. On note également, parmi les pétitionnaires malheureux, une surreprésentation des militaires et des marins ${ }^{16}$ qui témoigne de la diminution progressive des grandes expéditions scientifiques au cours du XIX ${ }^{\mathrm{e}}$ siècle $^{17}$. À l'inverse, les personnels des différentes facultés et des grands établissements scientifiques ainsi que les archivistes et les bibliothécaires apparaissent comme des candidats idéaux au voyage scientifique : ils ne constituent que 6,2\% des demandeurs de mission malheureux, et forment près de la moitié des voyageurs subventionnés par

16. 9,2\% des pétitionnaires malheureux sont des militaires ou des marins, contre seulement 2,5\% des chargés de mission subventionnés par le ministère.

17. Sylvain Venayre, Panorama du voyage (1780-1920). Mots, figures, pratiques, Paris, Les Belles Lettres, 2012, p. 223. 
le ministère ${ }^{18}$. Ces individus disposent souvent d'une instruction poussée et de l'appui des réseaux savants de la capitale, qui facilitent l'adhésion des membres de la commission.

La diversité des profils des demandeurs de missions reflète la notoriété de la commission, qui n'est pas limitée à la sphère savante parisienne et s'étend à toutes les catégories sociales. Si l'immense majorité des candidats manifestent une bonne maîtrise de l'expression écrite et des codes épistolaires, plusieurs missives sont rédigées dans un français approximatif qui laisse supposer un degré d'instruction inférieur. En 1889, François Boilot, qui réside à Clermont-Ferrand et vient de terminer son service militaire en Tunisie, envoie ainsi à la commission une lettre émaillée de fautes d'orthographe pour demander à faire partie d'une «mission d'exploiratoeur» en Afrique ou «dans tout autre payis $»^{19}$. Dépourvu de tout titre scientifique et sans projet précis, il précise au ministre que ce n'est pas pour son instruction qu'il désire être subventionné, mais plutôt pour «faire quelque voyage dans dé lointin paiyi».

\section{«AMour de la SCIENCE " ET « GOÛT DES VOYAGeS" : \\ LA FIGURE DU SAVANT EXPLORATEUR}

La moitié des demandes refusées par la commission concernent des projets de missions d'exploration, qui permettent aux candidats de concilier leur amour de la science et leur attrait pour les voyages lointains. Si l'exploration n'a pas été inventée au XIX ${ }^{\mathrm{e}}$ siècle, elle acquiert à cette époque une importance croissante. La conquête coloniale, qui s'accélère dans les années 1880 , fournit un cadre favorable à l'organisation des missions : les principaux centres de colonisation constituent des points de départ et de ravitaillement potentiels et les nouveaux territoires offrent aux appétits scientifiques un champ nouveau et stimulant. L'exploration peut se définir à la fois par son terrain (qui est lointain et mal connu ${ }^{20}$ ), par ses modalités (celles d'un voyage représenté comme éprouvant, voire dangereux) et par sa finalité qui est d'ordre scientifique, l'exploration ayant pour objectif principal de faire progresser les connaissances. La géographie est la première discipline concernée par l'exploration, mais l'explorateur du XIX ${ }^{\mathrm{e}}$ siècle, dans le sillage des voyageurs savants du siècle précédent, ne se contente pas de parcourir et de cartographier des espaces inconnus. Il s'attache aussi à étudier précisément les territoires qu'il visite, en pratiquant un large éventail de disciplines scientifiques (géologie, botanique, géographie, zoologie, ethnographie, etc.).

18. Selon les chiffres de Jean-Christophe Bourquin, les personnels des facultés représentaient $17,4 \%$ des chargés de mission, ceux des grands établissements scientifiques $21,3 \%$ et les archivistesbibliothécaires $9,6 \%$.

19. AN, dossier Boilot, F17 29401.

20. Les missions européennes ne sont que très rarement qualifiées de "missions d'exploration", l'appellation étant réservée aux destinations d'outre-mer. 
L'analyse des lettres de demandes de mission permet de dégager les ressorts de cet attrait pour l'exploration, qui constitue une modalité importante de la libido sciendi au XIX ${ }^{\mathrm{e}}$ siècle. Les destinations envisagées par les pétitionnaires confirment leur engouement pour les lointains et l'inconnu ${ }^{21}$. Ainsi, la moitié des demandes concernent l'Afrique, et en particulier les zones mal connues des Européens, les fameux «blancs de la carte»; l'Asie recueille près du quart des demandes de mission, et l'Amérique $18 \%$, avec une prédilection marquée pour les espaces vierges de l'Amazonie. Si les candidats à l'exploration savante professent invariablement leur amour de la science et leur goût pour les voyages, la proportion de chacune de ces motivations semble varier fortement en fonction des individus. Dans quelques cas, qui concernent généralement des savants professionnels bien insérés dans les réseaux académiques, le voyage lointain apparaît uniquement comme un moyen de parvenir à la connaissance. C'est le cas du botaniste Louis Crié, professeur à la faculté des sciences de Rennes et correspondant national de l'Académie de médecine, qui réclame en 1881 une mission botanique et géographique en Océanie afin d'étudier la "germination des microspores" du Phylloglossum Drummondi ${ }^{22}$. Il espère prouver «l'étroite affinité des Lycopodiacées isosporées et des Ophioglossées " et souligne avec enthousiasme "l'intérêt philosophique qui s'attache à un pareil document». Son itinéraire, qu'il présente en détail mais de manière sobre, est déterminé exclusivement par les spécimens qu'il souhaite étudier et auxquels il consacre la plus grande partie de sa lettre.

Dans bien des cas, cependant, ce sont le terrain de l'exploration et les modalités du voyage qui semblent déterminants dans les demandes des candidats : le voyage n'apparaît plus alors comme un moyen d'accéder à la connaissance scientifique mais comme une promesse d'aventures exotiques, qui constituent une fin en soi. La figure du savant cède ici le pas à celle de l'«aventurier», qu'on peut définir, avec Sylvain Venayre, comme «celui qui recherche les aventures pour elles-mêmes, à l'exclusion de tout autre but " ${ }^{23}$. L'évocation des territoires inconnus prend alors le pas sur celle des objectifs scientifiques. Certaines lettres peignent ainsi le décor fantastique de jungles luxuriantes, de cités englouties ou de trésors aztèques ${ }^{24}$. D'autres insistent longuement sur les modalités du voyage : c'est le cas, par exemple, d'une demande qu'envoie en 1893 un lieutenant d'infanterie de marine afin d'obtenir une mission d'exploration des régions situées entre la plaine d'Annam et le bassin du Mékong. Une partie importante de sa lettre, rédigée au condi-

21. Sylvain Venayre, La Gloire de l'aventure. Genèse d'une mystique moderne, 1850-1940, Paris, Les Belles Lettres, 2002, p. 46-51.

22. AN, dossier Crié (F17 2951).

23. Sylvain Venayre, "La Belle époque de l'aventure (1890-1920)", Revue d'histoire du XIXe siècle, $\mathrm{n}^{\circ} 24,2002$, p. 98.

24. AN, dossier Hérissez (F17 2975). 
tionnel, est consacrée à l'évocation prospective des difficultés et des obstacles qu'il pense rencontrer :

Les régions que je désire explorer sont habitées, en effet, par des tribus Moïs, Sendangs et Rodez qui semblent vouloir empêcher, même par la force, les voyageurs de pénétrer sur leur territoire. J'essaierais de me les concilier par mes dispositions bienveillantes et, accompagné d'une très faible escorte, d'en obtenir le passage à force de calme et de patience. Je n'aurais guère à compter sur le concours de ces habitants pour obtenir des vivres et des renseignements. Les chemins me manqueraient, sans doute parfois, faute de guides. Il faudrait me diriger alors à la boussole et me frayer un chemin au coupe-coupe. Les fièvres, les fatigues, les maladies de toutes sortes et les privations m'arrêteraient souvent. ${ }^{25}$

Dans cette lettre, envoyée depuis le petit village de Romery dans la Marne, qui abrite 175 habitants en $1906^{26}$, l'objectif scientifique, formulé en des termes très vagues, s'efface derrière un lointain de convention qui n'est pas sans rappeler les vignettes de la presse illustrée.

Celle-ci a joué en effet un rôle majeur dans la popularisation des explorateurs, en alimentant l'imaginaire du voyage lointain. Libérée des contraintes de la censure sous la Troisième République, elle connaît dans les dernières décennies du XIX ${ }^{\mathrm{e}}$ siècle un essor spectaculaire, qui accompagne et nourrit celui de la libido sciendi. La prestigieuse Société de Géographie de Paris publie dès les années 1820 des nouvelles des explorateurs français en mission outremer, détaille leurs itinéraires et les obstacles qu'ils rencontrent et édite leurs rapports ${ }^{27}$. Mais c'est à partir des années 1870 , dans un contexte européen de compétition coloniale qui atteint son apogée après la conférence de Berlin en 1884-1885, que l'explorateur devient une figure incontournable des pages de la presse généraliste illustrée ${ }^{28}$. De nombreux pétitionnaires suivent dans les colonnes des journaux les périples des explorateurs français, ce dont témoigne la chronologie des demandes. Ainsi, en 1880 et 1881, trois personnes demandent à être chargées de la mission interrompue par la mort de l'abbé Debaize ${ }^{29}$, dont ils ont pris connaissance par la presse. Véritable fiasco scientifique, cette mission avait reçu du Parlement, en 1878, la somme considérable de 100000 francs pour effectuer la traversée de l'Afrique d'Est en Ouest. Son organisateur, Michel Debaize, mourut d'une insolation sans laisser aucun document utile à la science ${ }^{30}$. Quatre ans plus tard, l'échec de Jules Crevaux, tué au cours de son voyage en Guyane ${ }^{31}$, suscite quatre demandes

25. AN, dossier Debay (F17 2953).

26. Source : site Cassini, http://cassini.ehess.fr.

27. Alfred Fierro, La Société de Géographie (1821-1946), Genève, Droz, 1983.

28. Felix Driver, Geography Militant. Cultures of Exploration and Empire, Oxford, Blackwell Publishers, 2001.

29. AN, dossiers L'Huissier (F17 2985 1), Moulin (F17 2994) et Slahf (F17 3007 1).

30. Emmanuelle Sibeud, Une science impériale pour l'Afrique?, op. cit., p. 22.

31. Francis Dupuy, "Deux explorateurs dans l'intérieur de la Guyane à la fin du XIX e siècle : 
visant à rechercher d'éventuels survivants de la mission ${ }^{32}$. À l'inverse, les succès de Pierre Savorgnan de Brazza ont fait beaucoup d'émules : ses méthodes pacifiques sont citées en exemple dans de nombreuses lettres de demandes de mission $^{33}$ et cinq pétitionnaires demandent à être adjoints ${ }^{34}$ au célèbre explorateur, dans les années 1880 . Cet engouement s'explique en grande partie par l'écho que rencontrent en métropole les réussites de Brazza: la presse française se passionne en effet pour le traité que l'explorateur est parvenu à signer avec le «roi Makoko» au Congo en septembre $1880^{35}$.

Les lettres des candidats à l'exploration révèlent, en creux, la manière dont les représentations diffusées par la presse et la littérature ${ }^{36}$ ont contribué à alimenter et à façonner la libido sciendi hors des cercles académiques. Les demandes sont ainsi profondément imprégnées de l'«imagerie nouvelle» diffusée par la presse, «qui donne à voir l'exploration en acte en présentant des scènes telles que les traversées de rivières, passages périlleux de rapides en canots, ou progressions difficiles en forêt dense, où l'on se fraie un chemin à la machette ${ }^{37}$. Les gravures du Journal des voyages ou du Tour $d u$ monde semblent avoir vivement frappé l'imagination des pétitionnaires. Jean Palmade, employé des chemins de fer, rappelle par exemple à la commission qu'il a été maitre de natation à l'école régimentaire et sait conduire des embarcations ${ }^{38}$; tandis que plusieurs candidats insistent sur leurs talents de chasseurs ${ }^{39}$. Les dangers de l'exploration sont largement soulignés dans la presse illustrée : le Journal des voyages publie ainsi le 12 novembre 1882 le récit de la mort du docteur Crevaux, accompagné d'une gravure qui le montre aux prises avec une dizaine d'Indiens Tobas armés de machettes ${ }^{40}$. Cette héroïsation de la figure de l'explorateur, élevé au rang de «martyr de la science $»^{41}$, ne constitue que l'une des modalités littéraires de la représentation du savant en héros, mises en évidence dans ce dossier par Denis SaintAmand ${ }^{42}$. Ces représentations expliquent que les pétitionnaires consacrent autant d'énergie à protester de leur courage et de leur sens du sacrifice auprès du ministère. Certains prennent même le soin de préciser qu'ils sont céli-

\footnotetext{
Jules Crevaux et Henri Coudreau", in Christiane Demeulenaere-Douyère (dir), Explorations et voyages scientifiques de l'Antiquité jusqu'à nos jours. Actes du $130^{\circ}$ Congrès national des sociétés historiques et scientifiques, La Rochelle, 2008, p. 159-181.

32. AN, dossiers Goujon (F17 2971), Guierre (F17 2973 2) et Louradour (F17 2985 2).

33. Voir notamment AN, dossier Leloire (F17 2983 2).

34. AN, dossiers Clauzet (F17 2949), Debay (F17 2953), Dusonchet (F17 2958 2), Habert (F17 2974 1), Vialla (F17 3013).

35. Elikia M'Bokolo, Afrique noire. Histoire et civilisations, du XIXe siècle à nos jours, Paris, Hatier, 2004, p. 277.

36. Matthieu Letourneux, Le roman d'aventures, 1870-1930, Limoges, PULIM, 2009.

37. Isabelle Surun, art. cit., p. 65.

38. AN, dossier Palmade (F17 2996 1).

39. AN, dossiers Habert (F17 2974 1) et Vialla (F17 3013).

40. Journal des voyages et des aventures de terre et de mer, 12 novembre 1882, n 279, p. 298-302.

41. Isabelle Surun, art. cit, p. 61.

42. Voir l'article de Denis Saint-Amand p. 107.
} 
bataires et ne possèdent aucun parent ${ }^{43}$. Outre la bravoure, la jeunesse et la force physique apparaissent dans les lettres des pétitionnaires comme deux qualités indispensables à l'apprenti explorateur : les candidats insistent sur leur "tempérament sec et nerveux ${ }^{44}$, sur leur «force musculaire peu commune ${ }^{45}$ et sur leur santé de fer ${ }^{46}$, et dissimulent soigneusement leur âge quand celui-ci constitue un obstacle ${ }^{47}$.

La presse et la littérature alimentent chez de nombreux pétitionnaires une véritable fascination pour les expéditions scientifiques, en dépit d'une absence totale d'expérience ou de qualifications. C'est le cas de Claude Dusonchet, qui n'a jamais quitté sa Savoie natale mais se dit «dominé par l'idée des expéditions africaines». Le portrait que dresse de lui le préfet de Savoie, dans une lettre confidentielle jointe au dossier, dessine les contours d'une vie de frustrations :

M. Dusonchet (Claude) est un jeune homme de 21 ans. Il fait partie de la classe 1882 et a été ajourné à un an par le conseil de révision pour défaut de taille (1 mètre 50); il est peu robuste et supporterait difficilement les fatigues d'une expédition sur le continent africain. D'un autre côté, il n'a reçu qu'une instruction primaire très restreinte; il a été pendant quelque temps clerc d'avoué et travaille actuellement chez un agent d'affaires de Chambéry. À une certaine époque, il a sollicité un emploi dans les bureaux de la Préfecture de la Savoie, mais sa mauvaise écriture et son orthographe défectueuse n'ont pas permis de prendre sa demande en considération. Dans ces conditions, Monsieur le Ministre, j'estime que $\mathrm{M}$. Dusonchet est absolument incapable de rendre des services quelconques à une mission scientifique. ${ }^{48}$

Le rôle de la commission consiste en partie à écarter ce type de demandes, lorsque l'enthousiasme ne suffit pas à suppléer aux qualifications.

\section{LA COMMISSION DES MISSIONS : INSTANCE DE LÉGITIMATION SCIENTIFIQUE}

De 1874 au milieu du XX $X^{\mathrm{e}}$ siècle, la commission des missions et des voyages scientifiques et littéraires a joué en France un rôle central dans le financement de projets scientifiques. Elle est l'interlocutrice principale des voyageurs à la recherche de subventions puisqu'elle dispose du budget

43. AN, dossier Lagrange (F17 2980 2).

44. AN, dossier Vialla (F17 3013).

45. AN, dossier Le Saint (F17 2984 2).

46. AN, dossier Lagrange (F17 2980 2).

47. Le docteur Sace est ainsi décrit comme «fort âgé et agité», par le rapporteur de son projet, AN, dossier de Sace (F17 3005 1).

48. AN, dossier Dusonchet (F17 2958 2). 
le plus important alloué aux voyages scientifiques : entre 1842 et 1914, le ministère de l'Instruction publique, dont elle dépend, a consacré plus de six millions de francs à de tels projets, pour une moyenne de 16,5 missions par $\mathrm{an}^{49}$. Les membres de la commission, dont le nombre varie entre 20 et 44 au fil des années, sont chargés d'examiner tous les projets de mission, de déterminer ceux auxquels il convient d'apporter une aide financière, et d'encadrer les voyageurs en leur procurant conseils et instructions. En sélectionnant, parmi les très nombreuses demandes, celles qui méritent l'attention du ministère, la commission fonctionne comme la principale interface entre l'État et les voyageurs savants, auxquels elle accorde ou refuse une légitimité scientifique matérialisée par le titre de chargé de mission. Des savants renommés disposant de positions institutionnelles prestigieuses (professeurs du Muséum d'histoire naturelle, du Collège de France, de l'École des langues orientales ou de l'École des Chartes, membres de l'Institut) y siègent aux côtés d'explorateurs reconnus (comme Alfred Grandidier, spécialiste de Madagascar, membre de la commission de 1891 à 1914). Le reste des sièges est occupé par des hommes politiques, des représentants de l'administration et quelques amateurs éclairés : la proportion de savants dans la commission est en moyenne de $31,5 \%$, celle des hommes politiques de $26,2 \%$, tandis que les représentants de l'administration et les amateurs occupent respectivement $33,1 \%$ et $7,7 \%$ des sièges ${ }^{50}$. La commission étudie l'intérêt scientifique du projet présenté, ainsi que les capacités du pétitionnaire à le mener à bien. Le travail d'analyse de la candidature est effectué par un membre de la commission, dont le profil correspond à la nature du projet présenté. Ainsi, entre 1879 et 1892, les demandes de mission pour le Sahara sont généralement examinées par Henri Duveyrier, qui s'est rendu célèbre par l'exploration de cette région. Le membre référent de la commission rédige un rapport, dont on fait la lecture lors de la réunion de la commission ${ }^{51}$. Cette dernière s'en remet généralement à l'avis du rapporteur.

Les dossiers refusés par la commission comprennent quasiment tous la copie de la réponse envoyée au candidat. Le motif du refus y est parfois précisé, mais on utilise souvent un courrier type et des formules stéréotypées (du type «il n'y a pas lieu d'accorder la demande», ou «la commission n'a pas cru devoir accepter votre proposition»). Si le manque de crédits est généralement invoqué pour justifier les refus, il ne s'agit pas toujours du principal facteur : on compte ainsi parmi l'échantillon 34 demandes de missions gratuites qui n'ont pas recueilli l'accord du ministère ${ }^{52}$. Le véritable motif du refus transparaît dans d'autres pièces conservées au dossier, et notamment

49. Jean-Christophe Bourquin, op. cit., p. 126.

50. Ibid., p. 87.

51. Voir les procès-verbaux de la commission des missions et voyages scientifiques et littéraires, aux AN : F17 2972, F17 2973 et F17 17225.

52. Il semble cependant qu'il s'agisse parfois du véritable motif, comme dans le cas de ClermontGanneau, dont la mission est refusée en 1886, malgré l'avis positif d'Ernest Renan (F17 2949). 
dans les rapports rédigés par les membres de la commission. Ces derniers permettent d'appréhender la manière dont se construisent, dans la deuxième moitié du XIX ${ }^{\mathrm{e}}$ siècle, les contours de la légitimité scientifique. L'un des principaux critères utilisés est le caractère désintéressé du projet : une mission est considérée comme désintéressée lorsque son objectif premier, et unique, est de faire avancer la connaissance. Sont ainsi écartées toutes les demandes qui relèvent de motivations troubles. En effet, pour certains demandeurs, la science ne constitue pas un objectif en soi mais paraît plutôt offrir la perspective d'un nouveau départ. Ces individus, qu'il s'agisse d'escrocs, d'imposteurs ou de personnages aux mœurs douteuses, paraissent vouloir utiliser les crédits du ministère de l'Instruction publique pour échapper aux autorités ou à leurs créanciers. Par mesure de précaution, la commission des missions demande souvent aux préfets, de manière confidentielle, des informations sur les pétitionnaires dont les noms lui sont inconnus, ce qui a permis au ministère de l'Instruction publique d'éviter plusieurs erreurs. Ainsi, parmi les 292 dossiers étudiés, on compte six demandes déposées par des escrocs ou des imposteurs, et trois demandes d'individus poursuivis par des créanciers ${ }^{53}$. Leur prétendu goût pour la science masque des motivations équivoques, que la commission met au jour en faisant appel à un réseau d'informateurs. En 1886, un individu qui se fait appeler le "père Aurélien» et se prétend curé de Magella en Italie et directeur apostolique d'un orphelinat agricole sollicite une mission en Palestine afin d'effectuer des recherches sur les premiers temps du christianisme ${ }^{54}$. Ernest Renan, dont il se recommande, envoie au ministère un entrefilet du Temps, daté 21 mars 1886, qui met en garde les lecteurs contre un "dangereux escroc» décrit comme un «homme de petite taille, aux cheveux grisonnants, à la physionomie intelligente et rusée». Dom Aurélien est en réalité un faiseur de dupes qui sillonne l'Europe. Après avoir monté plusieurs escroqueries en Espagne et en France, il est condamné à trois mois de prison, ce qui ne l'empêche pas de récidiver en 1885, à Lyon, où il fait imprimer une brochure sur "Les Illuminations du 8 décembre», qu'il vend à des acheteurs persuadés de faire une œuvre pieuse. Conformément à l'avis de Renan, le ministère refuse la demande de mission du père Aurélien.

Même lorsqu'il ne relève pas de l'escroquerie, l'appât du gain apparaît comme inconciliable avec le caractère scientifique d'une mission ${ }^{55}$. En 1893, Pelletier demande une mission en Indochine ${ }^{56}$ afin de préparer la fondation d'une "Société d'études pour créer et favoriser le commerce et l'industrie dans les contrées indépendantes de la vallée du Mékong». Le directeur de

53. AN, dossiers du père Aurélien (F17 2934 2), Jules Carrey (F17 2944), Paul l'Épine (F17 2984 2), Édouard Foa (F17 2962), Paul Perny (F17 2996 2) et Thomas Caraman (F17 3009 1).

54. AN, dossier du père Aurélien (F17 2934 2).

55. Sur la question du désintéressement nécessaire des hommes de science, $c f$. Steven Shapin, Une histoire sociale de la vérité. Science et mondanité dans l'Angleterre du XVII' siècle, Paris, La Découverte, 2014 (1994), p. 109.

56. AN, dossier Pelletier (F17 2996 2). 
l'École coloniale, Étienne Aymonier, rédige un rapport très défavorable à Pelletier, et souligne que "le caractère scientifique de sa mission est absolument accessoire", le but principal semblant être de chercher de l'or, du pétrole, du charbon de terre et de favoriser le commerce des bestiaux. Le désintéressement du pétitionnaire constitue, aux yeux de la commission, une garantie essentielle du caractère scientifique de la mission. Ainsi, les missions comportant une application pratique et lucrative, qu'elle soit industrielle ou commerciale, sont évaluées avec la plus grande méfiance. C'est ce qui explique, par exemple, que la demande de Carbonnel visant à étudier l'huître perlière dans le golfe de Panama, le golfe de Suez et la mer Rouge soit refusée à quatre reprises et renvoyée au ministère du Commerce ${ }^{57}$. Une vingtaine de demandes de ce type sont refusées ou renvoyées à d'autres ministères ${ }^{58}$.

Outre le caractère désintéressé du projet, la commission évalue les capacités du pétitionnaire à le mener à son terme. Pour ce faire, les membres de la commission prennent en compte la formation du demandeur, ses travaux antérieurs, ainsi que son inscription dans les réseaux savants, qui constitue à leurs yeux un gage de sérieux et de fiabilité. Cette procédure revêt une importance capitale car l'éloignement des terrains de recherche favorise l'autonomie du chargé de mission ${ }^{59}$. Si, comme le souligne Jean-Christophe Bourquin, les membres de la commission s'intéressent avant tout au projet de mission et à ses bénéfices éventuels pour la science, le parcours du pétitionnaire ne paraît pas aussi secondaire qu'il semble le suggérer lorsqu'il affirme que les pétitionnaires défendent «d'abord un projet de voyage, sans sentir la nécessité de l'étoffer par un curriculum vitae ${ }^{60}$. En effet, les dossiers de mission refusés mentionnent très souvent les titres scientifiques des demandeurs, parfois sous la forme d'épais fascicules reliés. Il ne s'agit pas là d'une précaution vaine, car de nombreux refus sont motivés par l'absence ou l'insuffisance des titres scientifiques des candidats. Les rapporteurs traquent également, dans les lettres de demande de mission, les erreurs factuelles qui trahiraient des lacunes : une méconnaissance des travaux récents peut ainsi entraîner un refus de la mission. La commission n'exclut pas systématiquement les demandes des amateurs, mais elle attend d'eux qu'ils comblent leurs lacunes en recueillant les conseils des professeurs du Muséum d'histoire naturelle et en suivant certains de leurs enseignements ${ }^{61}$. Les insuffisances des voyageurs seront ensuite partiellement neutralisées par l'utilisation d'instructions détaillées et de questionnaires élaborés en métropole, comme l'a montré Claude

57. AN, dossier Carbonnel (F17 2944).

58. Six candidats ont également déposé une demande de mission commerciale auprès du ministère du Commerce : AN, dossiers Dumas (F12 7410), Dutemple, Lacotte, Leloire (F12 7412), Largeau (F12 7212), Viard (F12 7413).

59. À propos de l'importance de la notion de confiance (trust) dans le cas de la science au loin, $c f$. David N. Livingstone, Putting Science in Its Place. Geographies of Knowledge, Chicago, The University of Chicago Press, 2003, p. 147 et suiv.

60. Jean-Christophe Bourquin, op. cit., p. 230.

61. AN, dossier Jouin (F17 2978). 
Blanckaert dans le cas des sciences de l'homme $e^{62}$. Par ailleurs, si l'affiliation à une société savante peut constituer un atout, elle ne suffit pas à pallier la non appartenance aux réseaux savants de la capitale. Ainsi, un pétitionnaire dont le nom est inconnu des membres de la commission a-t-il moins de chance d'obtenir une mission ${ }^{63}$.

Tout en se présentant comme un instrument de la démocratisation de l'accès à la science (puisque toutes les demandes sont en principe soumises à son examen), la commission des missions instaure en réalité des mécanismes de sélection qui marginalisent certaines catégories d'individus. Deux cas de figure sont particulièrement éloquents : celui des étrangers et celui des femmes. Le crédit des missions est réservé aux ressortissants français et un demandeur étranger se voit systématiquement refuser l'appui du ministère, sans égard pour ses qualifications scientifiques ou l'intérêt de son projet. Cinq demandes sont ainsi refusées pour ce motif ${ }^{64}$, ce qui s'explique en partie par le contexte de compétition scientifique internationale croissante. Certains pétitionnaires tentent d'utiliser cette concurrence à leur profit en faisant appel au patriotisme de la commission, voire en exerçant une forme de chantage (César Rousseau menace par exemple de se tourner vers le gouvernement anglais $\left.{ }^{65}\right)$. Par ailleurs, les demandes formulées par des femmes sont systématiquement refusées. En 1888, la demande de mission d'Anne Levinck est ainsi refusée au motif qu' «il n'est pas dans les usages du ministère de l'Instruction publique de confier une mission à une femme, lorsque cette femme n'a pas, avec le département, des attaches professionnelles ${ }^{66}$. Aucune explication supplémentaire n'est fournie pour justifier cette règle.

Les candidats au voyage scientifique mettent en œuvre des stratégies variées afin d'emporter l'assentiment de la commission. Beaucoup joignent à leur demande une longue liste de travaux (articles, ouvrages, et communications scientifiques) ainsi que des recommandations. Ces dernières peuvent prendre la forme d'une lettre adressée au ministère et annexée au dossier, d'une simple note manuscrite en marge de la demande, voire d'une carte de visite glissée dans l'enveloppe. Les recommandations les plus efficaces sont celles qui émanent d'autorités scientifiques, mais beaucoup de pétitionnaires joignent également des recommandations émanant de fonctionnaires ou d'hommes politiques. Le cas le plus remarquable est celui d'Albert Vayssière, préparateur de zoologie à la faculté des sciences de Marseille ${ }^{67}$, qui joint à sa demande de mission aux îles Seychelles pas moins de huit lettres de recommandation rédigées par différents députés, ce qui lui vaut un commentaire

62. Claude Blanckaert (dir), Le terrain des sciences humaines (XVIII'-XXe siècle), Paris, L'Harmattan, 1996.

63. AN, dossier Angeli (F17 2933 2) : «Le pétitionnaire m’est inconnu, même de nom ».

64. AN, dossiers Brzozowski (F17 2943 1), Debayle (F17 2953), Montet (F17 2993 1), SignoirSinnett (F17 3007 1) et Zeballos (F17 3014 2)

65. AN, dossier Rousseau (F17 3004 2).

66. AN, dossier Levinck (F17 2984 2).

67. AN, dossier Vayssière (F17 3012 1). 
acerbe du ministre ( un joli type d'intrigant ce $\mathrm{Mr}$ [sic], huit lettres de députés et combien de bonnes raisons?»). Aux recommandations s'ajoute souvent une forme de chantage politique. Sous la Troisième République, en effet, nombreux sont les pétitionnaires qui soulignent leur attachement ancien au régime républicain et leur opposition à l'Empire. Gaston Vialla, employé dans une administration, rappelle ainsi que son père "ex-conseiller municipal de Lasalle [...] s'est toujours fait remarquer par son opposition à l'Empire, et était en tête d'une liste de proscription dressée à la mairie de Lasalle quelques mois avant le quatre septembre ${ }^{68}$. Les lettres se teintent parfois d'une certaine aigreur, comme celle de monsieur Gazeau de Vautibault qui s'estime victime d'une injustice ("Y-a-t-il là malentendu, ou bien quelque réactionnaire, quelque clérical a-t-il voulu me nuire en ma qualité de républicain de vieille date et d'adversaire militant du cléricalisme?») ${ }^{69}$. L'insistance avec laquelle les candidats proclament leur allégeance au régime républicain est liée à la composition même de la commission. Héritière du service des missions, qui a survécu aux changements de régime successifs, la commission, créée officiellement en 1874, a été réorganisée afin d'intégrer des personnalités soigneusement sélectionnées par le ministère de l'Instruction publique : républicains convaincus, largement gagnés à l'anticléricalisme, tous les membres (à quelques rares exceptions près) disposent d'une position solide au sein des institutions politiques et académiques de la Troisième République. La science apparaît d'ailleurs, sous leur plume comme dans les lettres des pétitionnaires, comme une entreprise patriotique : de nombreux candidats se disent ainsi mus par le désir d'être «utiles à leur pays » ${ }^{70}$.

*

Les dossiers des pétitionnaires malheureux de la section F17 donnent à voir la manière dont fonctionne, au niveau individuel, le désir de savoir qui s'empare de la société française au XIX ${ }^{\mathrm{e}}$ siècle à travers l'une de ses modalités : le voyage scientifique d'exploration. Les lettres qu'ils renferment émanent pour la plupart d'individus dont l'histoire n'a pas retenu le nom et livrent des détails précieux sur les parcours et les motivations de ces candidats au voyage scientifique lointain. Dans la deuxième moitié du XIX siècle s'élabore un modèle du voyage scientifique d'exploration dont la légitimité repose largement sur son caractère désintéressé, et qui se distingue d'autres formes de mobilités individuelles, comme l'aventure, popularisée par le genre romanesque à partir des années 1890 , ou le simple voyage, rendu possible par la révolution des transports et l'extension des loisirs. Les candidats malheureux

68. AN, dossier Vialla (F17 3013).

69. AN, dossier Vautibault (F17 2970 1).

70. La formule revient notamment dans les dossiers de Morisot (AN, F17 2994), Besançon (AN, F17 2939), et Vialla (AN, F17 3013). 
du ministère de l'Instruction publique sont parfaitement conscients de ces enjeux de définition et tentent d'emporter l'adhésion de la commission en affirmant leur amour inconditionnel de la science. Transparaît néanmoins, entre les lignes, l'attrait immense qu'exercent sur eux non seulement les modalités de l'exploration, qui conferent à l'aventure au loin la respectabilité de l'entreprise scientifique, mais également la figure héroïsée de l'explorateur, popularisée par la presse et la littérature, qui va jusqu'à susciter des vocations chez des individus dénués de toute formation scientifique, voire d'instruction. Le patient travail d'examen et de renseignement mené par les membres de la commission des missions, dont les dossiers gardent les traces comme autant de pièces à conviction, permet aussi de retracer des parcours personnels et professionnels semés d'échecs et de déceptions, parfois entachés par le scandale. Les voyages d'exploration constituent ainsi, dans bien des cas, une occasion de fuir une existence morne ou difficile, et de trouver au loin la reconnaissance qu'on peine à obtenir chez soi. En refusant ces demandes, la commission des missions participe à la construction d'un nouveau modèle de légitimité scientifique qui émerge dans la deuxième moitié du XIXe siècle.

Stéphanie Soubrier est doctorante à l'Université Paris 1 Panthéon-Sorbonne et membre du Centre d'histoire du XIXe siècle 